Élido Bonomo ${ }^{1}$

Waleska Teixeira Caiaffa 2

Cibele Comini César 3

Aline Cristine Souza Lopes 4

Maria Fernanda Lima-Costa 2,5

\title{
Consumo alimentar da população adulta segundo perfil sócio-econômico e demográfico: Projeto Bambuí
}

\author{
Food intake according to socioeconomic \\ and demographic profile: the Bambuí Project
}

1 Departamento de Nutrição Clínica e Social, Escola de Nutrição, Universidade Federal de Ouro Preto. Campus Universitário, Morro do Cruzeiro, Ouro Preto, $M G$ 35400-000, Brasil.

ebonomo@enut.ufop.br

2 Departamento de Medicina Preventiva e Social, Faculdade de Medicina, Universidade Federal de Minas Gerais. Av. Alfredo Balena 190, Belo Horizonte, $M G$ 30130-100, Brasil.

3 Departamento de Estatística, Instituto de Ciências Exatas, Universidade Federal de Minas Gerais. Av. Antônio Carlos 6627, Belo Horizonte, $M G$ 31270-901, Brasil.

${ }^{4}$ Faculdade de Ciências Biológicas e da Saúde, Unicentro Newton Paiva. Rua Goitacases 1762, Belo Horizonte MG 30190-052, Brasil. 5 Centro de Pesquisa René Rachou, Fundação Oswaldo Cruz. Av. Augusto de Lima 1715, Belo Horizonte, $M G$ 30190-002, Brasil.

\begin{abstract}
A nutritional survey was performed in a random sample of 546 individuals (ages 18 and over) in a city named Bambuí (15,000 inhabitants) in Minas Gerais State, Brazil, using the Semi-Quantitative Food Frequency Questionnaire (SFFQ). Median calorie intakes for women and men were 2,807 and 3,775kcal, respectively. Men consumed four times more alcohol than women, and women consumed more carbohydrates, fiber, and polyunsaturated fatty acids (PUFA). The relative consumption of proteins (15\%), carbohydrates (57\%), and lipids (28\%) were adequate in both genders. The average proportions, for all participants, of an inadequate share of lipids, saturated fatty acids (SFA), PUFA, and cholesterol in the total calorie intake were, respectively, 36\%, 90\%, and 50\%, and were more pronounced in men than in women. The lipid, SFA, and PUFA intake for women and the lipid and SFA intake for men increased with income. The PUFA/SFA ratio and the PUFA and dietary fiber intake were below the recommended levels in both genders. Dietary habits presented a differential inadequacy, suggesting possible population risk clusters for cardiovascular diseases.
\end{abstract}

Key words Food Consumption; Nutrition Surveys; Diet

Resumo Foi realizado inquérito nutricional em amostra probabilística de 546 indivíduos (idade $\geq 18$ anos), residentes na cidade de Bambuí (15 mil habitantes), Minas Gerais, Brasil, utilizando-se o Questionário Semiquantitativo de Freqüência Alimentar (QSFA). A mediana de ingestão calórica entre mulheres e homens foi de 2.807 e $3.775 \mathrm{kcal}$, respectivamente. Homens ingeriram quatro vezes mais álcool que mulheres e estas, mais carboidratos, fibras e ácidos graxos polinsaturados (AGP). A distribuição de proteínas (15\%), carboidratos (57\%) e lipídios (28\%) foi adequada. As proporções médias de inadequação da participação de lipídios, ácidos graxos saturados (AGS), AGP e colesterol na energia total foram, respectivamente, 36\%, 50\% e 90\%, sendo mais pronunciadas entre os homens. A ingestão de lipídios, AGS e AGP, entre mulheres, e de lipídios e AGS entre homens, aumentou de acordo com a renda. A ingestão de AGP e fibras e a relação AGP/AGS estavam abaixo do recomendado nos dois gêneros. A dieta apresentou proporções de inadequação diferenciais, sugerindo possíveis agregados de risco à saúde da população para doenças cardiovasculares.

Palavras-chave Consumo de Alimentos; Inquérito Nutricional; Dieta 


\section{Introdução}

Os problemas decorrentes do consumo inadequado de alimentos já são conhecidos há muito tempo, expondo a população a graves e flagrantes danos à saúde, principalmente se considerarmos os diferenciais regionais existentes dentro e entre países (Drewnowski \& Popkin, 1997; Fung et al., 2001; Galeazzi et al., 1997; Monteiro et al., 2000; Pryer et al., 2001b). No entanto, apenas no final da primeira metade do século passado a epidemiologia da nutrição tomou impulso, e os primeiros métodos de avaliação nutricional com base populacional foram desenvolvidos (Willett, 1998). Recentemente, estudos observacionais têm evidenciado estreita relação entre características qualitativas da dieta e ocorrência de enfermidades crônico-degenerativas como as doenças cardiovasculares, diabetes mellitus não-insulinodependente, câncer e obesidade. Todas essas patologias constituem, hoje, um problema prioritário de saúde pública e de segurança alimentar, tanto em países desenvolvidos quanto naqueles menos desenvolvidos, incluindo todas as faixas etárias, com destaque entre crianças e adolescentes (Monteiro et al., 2000; Oliveira, 1993; OMS, 1990; Valente, 1997).

O perfil alimentar da população está fortemente associado a aspectos culturais, nutricionais, sócio-econômicos e demográficos, tornando necessário um melhor entendimento destes aspectos e seus mecanismos no entendimento das mudanças de comportamento alimentar e suas conseqüências (Kushi et al., 1988; Martins et al., 1994; Monteiro et al., 2000; Shimakawa et al., 1994; Thompson et al., 1992; van Horn et al., 1991).

Inquéritos dietéticos constituem o melhor instrumento para se determinar o padrão alimentar da população e a sua evolução com o tempo. No Brasil, via de regra, esses inquéritos são conduzidos em capitais e regiões metropolitanas. Desta forma, o padrão alimentar do interior do país é praticamente desconhecido. A mais completa fonte de informação alimentar com amostra representativa do país foi o Estudo Nacional de Despesa Familiar (ENDEF), conduzido em 1974/1975, com enfoque principal no consumo alimentar por meio da pesagem direta dos alimentos aplicados por sete dias consecutivos. Há outros estudos de abrangência nacional, como a Pesquisa Domiciliar de Orçamento Familiar (POF), realizada entre 1961 e 1963 em áreas urbanas e rurais; a POF realizada entre 1987 e 1988 em áreas metropolitanas e a POF realizada entre 1995 e 1996 em áreas metropolitanas e capitais brasileiras. Essas pesqui- sas estimaram o consumo alimentar médio per capita diário das famílias. Mais recentemente, destaca-se o Estudo Multicêntrico sobre o Consumo Alimentar e Estado Nutricional, realizado em 1996, por meio do qual foi examinado o consumo alimentar em cinco cidades brasileiras, sendo o primeiro estudo de grande abrangência no Brasil a utilizar o Questionário Semiquantitativo de Freqüencia Alimentar (QSFA) (Galeazzi et al., 1997; Monteiro et al., 2000).

Diversos instrumentos têm sido utilizados em inquéritos nutricionais. O QSFA, ainda pouco utilizado no Brasil (Sichieri \& Everhart, 1998), possibilita a avaliação da ingestão pregressa, com quantificação do tamanho das porções habituais, incluindo variações sazonais. Esse instrumento tem-se mostrado de grande utilidade para a investigação dos efeitos da dieta sobre a saúde (Pereira \& Koifman, 1999). Permite a investigação do padrão dietético em populações, fornecendo perfis alimentares e nutricionais fidedignos, subsidiando o planejamento das ações de saúde, possibilitando o estabelecimento de padrões regionais, úteis para estudos comparativos com outros grupos populacionais.

Este trabalho tem por objetivo conhecer o perfil alimentar, em termos de ingestão absoluta de nutrientes e suas participações relativas na energia total da dieta da população, identificando o potencial de proteção ou risco da dieta para doenças crônico-degenerativas, especialmente as cardiovasculares. As diferenças culturais, educacionais, sociais e econômicas da população brasileira, caracterizadas por fortes influências regionais - que podem influenciar o padrão da ingestão alimentar - motivaram a realização deste trabalho.

\section{Método}

\section{Projeto Bambuí}

O Projeto Bambuí é um estudo epidemiológico com base populacional desenvolvido na cidade de Bambuí, situada na região centro-oeste do Estado de Minas Gerais, com cerca de 15 mil habitantes. Esse projeto tem dois componentes: inquérito de saúde da população geral e estudo de coorte da população idosa (Lima-Costa et al., 2000). O presente trabalho faz parte do inquérito de saúde.

\section{Amostra do estudo}

Os participantes do estudo foram identificados por intermédio de censo realizado pela equipe 
do Projeto Bambuí, no período de outubro a dezembro de 1994. O inquérito de saúde foi realizado em uma amostra probabilística simples, sem reposição, de 1.664 moradores com 5 ou mais anos de idade (Lima-Costa et al., 2000). Entre os 1.221 participantes da amostra com 18 ou mais anos de idade, 660 foram aleatoriamente selecionados para o inquérito alimentar. A amostra final foi de 546 participantes. Maiores detalhes podem ser obtidos em trabalho anterior (Lopes, 1999).

\section{Coleta de dados}

As informações sobre a ingestão alimentar referentes aos últimos doze meses foram obtidas por meio do QSFA (Sichieri \& Everhart, 1998). O questionário continha informações sobre 94 alimentos. Foi utilizado um álbum de fotografias para minimizar o viés de memória e melhorar a qualidade da informação sobre o tamanho das porções servidas (Lopes, 1999). As entrevistas, realizadas face a face no domicílio, mediante consentimento prévio dos participantes, tiveram duração média de quarenta minutos. Os entrevistadores, selecionados entre residentes da cidade, tinham pelo menos o segundo grau completo e foram devidamente treinados para a pesquisa (Lima-Costa et al., 2000; Lopes, 1999).

As informações sócio-demográficas foram obtidas por intermédio da entrevista geral do Projeto Bambuí, utilizando perguntas estruturadas e pré-codificadas, incluindo as seguintes características: gênero, idade, escolaridade em anos completos concluídos e renda familiar em salários mínimos (SM) da época (Lima-Costa et al., 2000).

\section{Análise de dados}

A tradução das informações do QSFA em ingestão diária de nutrientes foi realizada por um programa criado para esse fim por meio do programa SPSS 11.0. Esse programa relaciona a quantidade ingerida com o valor nutricional do alimento, totalizando a ingestão diária por nutriente. A composição nutricional de cada alimento foi obtida por intermédio do banco de dados do Programa de Apoio à Nutrição, desenvolvido pela Escola Paulista de Medicina, conforme descrito por Lopes (1999). Para os alimentos não contemplados no banco, foram usadas as tabelas para avaliação de consumo alimentar em medidas caseiras (Pinheiro et al., 1994), e a tabela de composição química de alimentos (Franco, 1995).

Para avaliar diferenças na composição da dieta, a ingestão dietética foi ajustada pelo total de energia ingerida usando o método de proporção ou densidade calórica, exceto para fibras e colesterol, que foram ajustados por $1.000 \mathrm{kcal}$. Vale ressaltar que, a participação relativa dos macronutrientes e álcool não necessariamente totaliza $100 \%$ das calorias totais, levando em consideração o encontro de variados valores para o mesmo alimento nas tabelas de composição utilizadas (Burgos et al., 1996; Philippi et al., 1995). O ajustamento pela ingestão total de energia é apropriado em estudos epidemiológicos para controle de fatores de confusão (Willett et al., 1997). A relação entre ácidos graxos polinsaturados e ácidos graxos saturados (AGP/ AGS) foi incluída na análise. O consumo de álcool foi analisado considerando o porcentual de usuários e a ingestão mediana entre eles. Todas as análises foram estratificadas por gênero, faixa etária, renda e escolaridade.

Para a análise dos dados foram excluídas as informações dos entrevistados cujas ingestões diárias de energia e nutrientes estivessem abaixo do primeiro ou acima do 99o percentil, estratificadas por gênero. Dessa forma, a amostra do estudo variou para cada nutriente.

Optou-se pelos testes não paramétricos devido à presença de valores extremos, mesmo após as exclusões realizadas, e à grande assimetria da distribuição de alguns nutrientes. Os dados foram descritos utilizando-se a mediana e o desvio interquartílico, adotado como uma medida de variabilidade adequada para situações em que o centro é definido com base na mediana. O desvio interquartílico é a diferença entre o 3o e o 1o quartis (Soares \& Siqueira, 1999). O teste de Mann-Whitney foi utilizado para comparação de dois grupos independentes, e o de Kruskal-Wallis para mais de dois grupos. Para comparação de proporções de usuários de álcool foi utilizado o teste do quiquadrado $\left(\chi^{2}\right)$. Adotou-se o nível de significância de 0,05 .

O Projeto Bambuí foi aprovado pelo Comitê de Ética da Fundação Oswaldo Cruz (FIOCRUZ), em 1996.

\section{Resultados}

\section{Perfil sócio-econômico e demográfico} da amostra do inquérito alimentar

A amostra ( $\mathrm{n}=546)$, composta por $55,7 \%$ de mulheres e $44,3 \%$ de homens, foi classificada em quatro grupos etários: $18-30$ anos $(31,7 \%)$, $31-40(22,2 \%), 41-60(32,1 \%)$ e 60 anos ou mais $(14,1 \%)$. A média e desvio padrão e a mediana de idade foram, respectivamente, 41,6 \pm 16,4 e 
39,0 anos. Não houve diferenças significativas na distribuição etária e de gênero entre as amostras do inquérito de saúde e do alimentar.

No que se refere à renda familiar, 141 (25,8\%), 293 (53,7\%) e 112 (20,5\%) dos participantes recebiam, respectivamente, até 1,5 SM, de 3 a 5 e mais de 8 SM; nenhum indivíduo foi encontrado nas faixas intermediárias. Quanto à escolaridade, 295 (54\%), 108 (19,8\%) e 143 $(26,2 \%)$ tinham, respectivamente, até 4,5 a 8 e mais de 8 anos de estudo. Com o aumento da idade foi observado uma redução nas proporções de indivíduos nas faixas maiores de renda e escolaridade, e mais nas mulheres que nos homens (Tabela 1).

\section{Ingestão diária por gênero}

Na Tabela 2, estão apresentadas as medianas de consumo de fibras e de colesterol ajustados por $1.000 \mathrm{kcal}$ e o consumo dos demais nutrientes ajustado como proporção da energia total. A energia ingerida ( $\mathrm{p}<0,001)$, o percentual de usuários de álcool ( $\mathrm{p}<0,001)$ e a participação relativa de álcool $(\mathrm{p}<0,001)$ na energia total foram significativamente maiores entre os homens que entre as mulheres. A ingestão de álcool foi de $0,3 \%$ das calorias totais entre mulheres e $1,4 \%$ entre homens, sendo que $55 \%$ das mulheres e $69 \%$ dos homens relataram ter in- gerido álcool nos últimos 12 meses. As mulheres apresentaram maior ingestão de carboidratos $(\mathrm{p}=0,001)$, AGP $(\mathrm{p}=0,003)$ e fibras $(\mathrm{p}<$ $0,001)$, assim como maior relação AGP/AGS ( $\mathrm{p}=$ $0,03)$.

\section{Ingestão diária por gênero e idade}

Como pode ser observada na Tabela 3, a ingestão de energia total foi significativamente diferente entre as idades $(\mathrm{p}<0,001$ para mulheres $\mathrm{e}$ $\mathrm{p}=0,022$ para homens). Em ambos os gêneros percebe-se uma tendência de decréscimo da ingestão com o aumento da idade. Entre mulheres, as ingestões de álcool ( $\mathrm{p}<0,001)$, fibras $(\mathrm{p}=$ $0,011)$ e a proporção de usuárias de álcool $(\mathrm{p}<$ $0,001)$ foram diferentes por idade, ocorrendo menores consumos nas mulheres acima de 60 anos. Entre os homens, a ingestão de proteínas variou significativamente nos diferentes grupos etários ( $\mathrm{p}=0,022)$ e a relação AGP/AGS apresentou uma tendência de queda com o aumento da idade, sendo esta explicada pela variação observada no consumo de AGS. Embora não tenha sido observada diferença significativa $(p=0,060)$, o valor observado para o consumo de AGS entre homens com mais de 60 anos foi superior ao consumo nos outros grupos etários. Com relação ao álcool, maior proporção de usuários homens que mulheres foi observada $(\mathrm{p}<0,001)$.

Tabela 1

Distribuição da amostra do inquérito nutricional por renda e escolaridade,

de acordo com gênero e faixa etária. Município de Bambuí, Minas Gerais, Brasil, 1996-1997.

\begin{tabular}{|c|c|c|c|c|c|c|c|}
\hline \multirow[t]{2}{*}{$\begin{array}{l}\text { Características } \\
\text { sócio-demográficas }\end{array}$} & \multirow[t]{2}{*}{$n$} & \multicolumn{3}{|c|}{$\begin{array}{l}\text { Renda familiar em } \\
\text { salários mínimos (\%) }\end{array}$} & \multicolumn{3}{|c|}{$\begin{array}{l}\text { Escolaridade em } \\
\text { anos de estudo (\%) }\end{array}$} \\
\hline & & $\leq 1,5$ & $3-5$ & $\geq 8$ & $\leq 4$ & $5-8$ & $>8$ \\
\hline Inquérito nutricional & 546 & 25,8 & 53,7 & 20,5 & 54,0 & 19,8 & 26,2 \\
\hline \multicolumn{8}{|l|}{ Idade (em anos) } \\
\hline $18-30$ & 87 & 23,0 & 55,2 & 21,8 & 28,7 & 25,3 & 46,0 \\
\hline $31-40$ & 74 & 28,4 & 59,5 & 12,1 & 32,4 & 32,4 & 35,2 \\
\hline $41-60$ & 100 & 27,0 & 54,0 & 19,0 & 74,0 & 9,0 & 17,0 \\
\hline $60+$ & 43 & 53,5 & 39,5 & 7,0 & 93,0 & 4,7 & 2,3 \\
\hline Total & 304 & 29,9 & 53,6 & 16,5 & 53,6 & 18,8 & 27,6 \\
\hline \multicolumn{8}{|l|}{ Masculino } \\
\hline $18-30$ & 86 & 14,0 & 55,8 & 30,2 & 34,9 & 32,6 & 32,5 \\
\hline $31-40$ & 47 & 19,1 & 59,6 & 21,3 & 38,3 & 29,8 & 31,9 \\
\hline $41-60$ & 75 & 21,3 & 52,0 & 26,7 & 72,0 & 10,7 & 17,3 \\
\hline $60+$ & 34 & 38,2 & 44,1 & 17,7 & 88,2 & 2,9 & 8,9 \\
\hline Total & 242 & 20,7 & 53,7 & 25,6 & 54,5 & 21,1 & 24,4 \\
\hline
\end{tabular}


Ingestão diária de nutrientes selecionados (mediana e desvio interquartílico), em proporção das calorias totais e percentual de usuários de álcool, segundo gênero. Município de Bambuí, Minas Gerais, Brasil, 1996-1997.

\begin{tabular}{|c|c|c|c|}
\hline Variáveis dietéticas & Feminino $(n=304)$ & Masculino $(n=242)$ & $P^{*}$ \\
\hline Energia (kcal) & $2.807,4 \pm 1.537,8$ & $3.774,9 \pm 2.183,9$ & $<0,001$ \\
\hline Carboidratos (\% kcal) & $58,9 \pm 12,9$ & $55,9 \pm 15,1$ & 0,001 \\
\hline Proteínas (\% kcal) & $14,8 \pm 3,2$ & $14,9 \pm 3,6$ & 0,496 \\
\hline Lipídios (\% kcal) & $27,2 \pm 10,2$ & $27,4 \pm 12,4$ & 0,720 \\
\hline AGS (\% kcal) & $8,8 \pm 3,9$ & $8,9 \pm 4,5$ & 0,489 \\
\hline AGP (\% kcal) & $1,8 \pm 1,1$ & $1,6 \pm 0,9$ & 0,003 \\
\hline AGM (\% kcal) & $3,9 \pm 2,7$ & $3,9 \pm 2,6$ & 0,620 \\
\hline \multicolumn{4}{|l|}{ Álcool (\%) } \\
\hline Usuários & 54,9 & 69,4 & $<0,001$ \\
\hline Kcal** & $0,3 \pm 0,8$ & $1,4 \pm 3,4$ & $<0,001$ \\
\hline Colesterol (mg/1.000kcal) & $99,3 \pm 57,6$ & $102,8 \pm 60,5$ & 0,063 \\
\hline Fibras (g/1.000kcal) & $3,2 \pm 2,8$ & $2,3 \pm 1,9$ & $<0,001$ \\
\hline AGP/AGS & $0,22 \pm 0,13$ & $0,20 \pm 0,15$ & 0,030 \\
\hline
\end{tabular}

AGS = ácidos graxos saturados; $A G P=$ ácidos graxos polinsaturados; $A G M=$ ácidos graxos monoinsaturados * Valor $p$ - teste de Mann-Whitney para duas amostras independentes.

** A ingestão do álcool é apresentada entre os entrevistados que consumiram álcool.

Para os demais nutrientes, não foi observada variação significativa em ambos os gêneros.

\section{Ingestão diária por gênero e renda}

Na Tabela 4, verifica-se que as mulheres apresentaram diferenças significativas nas proporções ingeridas de carboidratos $(p=0,034)$, lipí$\operatorname{dios}(\mathrm{p}=0,026)$, AGS $(\mathrm{p}=0,001)$, AGP $(\mathrm{p}=$ $0,005)$, ácidos graxos monoinsaturados ( $\mathrm{p}=$ $0,049$ ) e colesterol ( $p=0,037)$ entre os distintos níveis de renda. Exceto para carboidratos e AGS, percebeu-se uma tendência de elevação do consumo desses nutrientes com o aumento do poder aquisitivo. A ingestão de AGS declinou na faixa de renda intermediária, seguida de aumento entre aquelas de maior poder aquisitivo. Entre os homens, o consumo de lipídios ( $\mathrm{p}=$ $0,031)$, AGS $(\mathrm{p}=0,053)$ e AGP $(\mathrm{p}=0,018)$ variaram significativamente nos distintos níveis de renda, apresentando redução no nível intermediário, seguido pelo aumento de ingestão na maior faixa de renda. Observa-se, também, elevação significativa da proporção de usuários de álcool com o aumento da renda, em ambos os gêneros $(\mathrm{p}<0,001)$.

\section{Ingestão diária por gênero e escolaridade}

Considerando os níveis de escolaridade (Tabela 5), observou-se que as mulheres apresentaram diferenças significativas de consumo energético $(\mathrm{p}=0,004)$, protéico $(\mathrm{p}=0,010)$ e AGP $(\mathrm{p}=0,009)$.
Entre os homens, nenhuma diferença significativa na ingestão foi observada. Mais uma vez, observa-se elevação significativa da proporção de usuários de álcool com o aumento da escolaridade, em ambos os gêneros $(\mathrm{p}<0,001)$.

\section{Discussão}

Neste estudo de base populacional, a dieta mostrou-se hipercalórica, normoprotéica, normo a hiperlipídica, normoglicídica, hipercolesterolêmica, rica em gorduras saturadas e pobres em gorduras polinsaturadas e fibras, com valores absolutos sempre maiores em homens que em mulheres. A relação AGP/AGS foi inferior a 0,70 para todos os participantes, sendo observada uma razão de 0,21 para a mediana e menores valores entre homens e idosos, sugerindo um pior prognóstico para doenças cardiovasculares nessa população.

Outras associações de importância expressiva para a saúde pública foram observadas de acordo com gênero, idade, renda familiar e escolaridade. Mulheres relataram ingerir proporcionalmente alimentos mais ricos em carboidratos, AGP e fibras quando comparadas aos homens. Estes, por sua vez, relataram ingerir alimentos mais ricos em energia e proporcionalmente mais álcool. Entre participantes com mais de 60 anos, as mulheres ingeriram menos fibras e álcool, enquanto os homens ingeriram mais proteínas. 
Ingestão diária de nutrientes selecionados (mediana e desvio interquartílico), em proporção das calorias totais, de acordo com idade e gênero. Município de Bambuí, Minas Gerais, Brasil, 1996-1997.

\begin{tabular}{|c|c|c|c|c|c|}
\hline \multirow[t]{2}{*}{ Variáveis dietéticas } & \multicolumn{4}{|c|}{ Idade (em anos) } & \multirow[b]{2}{*}{$P^{*}$} \\
\hline & $18-30$ & $31-40$ & $41-60$ & $61-92$ & \\
\hline Mulheres $(n=304)$ & $\mathrm{n}=87$ & $n=74$ & $n=100$ & $n=43$ & \\
\hline Energia (kcal) & $3.101,4 \pm 1.610,5$ & $2.855,5 \pm 1.322,4$ & $2.416,2 \pm 1.463,1$ & $2.674,0 \pm 1.566,9$ & $<0,01$ \\
\hline Carboidratos (\% kcal) & $56,7 \pm 12,9$ & $59,6 \pm 11,8$ & $60,2 \pm 11,6$ & $58,6 \pm 20,0$ & 0,223 \\
\hline Proteínas (\% kcal) & $14,9 \pm 3,1$ & $14,5 \pm 3,3$ & $14,5 \pm 2,9$ & $15,6 \pm 3,3$ & 0,060 \\
\hline Lipídios (\% kcal) & $29,2 \pm 10,2$ & $26,9 \pm 9,6$ & $26,5 \pm 10,3$ & $27,0 \pm 14,9$ & 0,261 \\
\hline AGS (\% kcal) & $9,1 \pm 3,9$ & $8,6 \pm 3,4$ & $8,8 \pm 3,7$ & $8,9 \pm 6,3$ & 0,665 \\
\hline AGP (\% kcal) & $1,8 \pm 1,0$ & $1,9 \pm 0,9$ & $1,8 \pm 1,0$ & $1,7 \pm 1,0$ & 0,397 \\
\hline AGM (\% kcal) & $4,2 \pm 2,8$ & $3,6 \pm 2,3$ & $3,6 \pm 2,6$ & $3,9 \pm 2,9$ & 0,467 \\
\hline \multicolumn{6}{|l|}{ Álcool (\%) } \\
\hline Usuárias & 70 & 72 & 40 & 30 & $<0,001$ \\
\hline Kcal ${ }^{* *}$ & $0,4 \pm 1,1$ & $0,3 \pm 0,8$ & $0,3 \pm 0,7$ & $0,1 \pm 0,2$ & $<0,001$ \\
\hline Colesterol (mg/1.000kcal) & $112,6 \pm 55,8$ & $101,7 \pm 54,5$ & $88,0 \pm 45,5$ & $101,0 \pm 81,5$ & 0,253 \\
\hline Fibras (g/1.000kcal) & $3,1 \pm 2,7$ & $3,9 \pm 3,5$ & $3,1 \pm 2,6$ & $2,8 \pm 2,3$ & 0,011 \\
\hline AGP/AGS & $0,22 \pm 0,10$ & $0,23 \pm 0,13$ & $0,22 \pm 0,15$ & $0,19 \pm 0,17$ & 0,842 \\
\hline Homens $(n=242)$ & $\mathrm{n}=86$ & $\mathrm{n}=47$ & $\mathrm{n}=75$ & $n=34$ & \\
\hline Energia (kcal) & $4.062,8 \pm 2.504,0$ & $3.524,2 \pm 1.748,0$ & $3.747,6 \pm 2.978,5$ & $3.155,8 \pm 2.075,7$ & 0,022 \\
\hline Carboidratos (\% kcal) & $55,3 \pm 13,7$ & $54,5 \pm 14,5$ & $56,6 \pm 15,1$ & $53,5 \pm 19,7$ & 0,958 \\
\hline Proteínas (\% kcal) & $14,9 \pm 3,3$ & $14,3 \pm 3,7$ & $14,2 \pm 3,3$ & $16,8 \pm 3,9$ & 0,022 \\
\hline Lipídios (\% kcal) & $28,1 \pm 12,9$ & $25,7 \pm 12,1$ & $27,112,2$ & $28,1 \pm 12,1$ & 0,568 \\
\hline AGS (\% kcal) & $9,0 \pm 4,6$ & $8,3 \pm 4,5$ & $8,7 \pm 4,7$ & $10,2 \pm 4,8$ & 0,060 \\
\hline AGP (\% kcal) & $1,7 \pm 1,0$ & $1,6 \pm 0,8$ & $1,5 \pm 0,9$ & $1,5 \pm 0,9$ & 0,311 \\
\hline AGM (\% kcal) & $4,0 \pm 2,5$ & $3,6 \pm 2,2$ & $3,9 \pm 2,8$ & $4,4 \pm 2,6$ & 0,652 \\
\hline \multicolumn{6}{|l|}{ Álcool (\%) } \\
\hline Usuários & 80 & 77 & 69 & 32 & $<0,001$ \\
\hline Kcal ${ }^{* \star}$ & $1,7 \pm 3,7$ & $1,4 \pm 5,0$ & $1,2 \pm 2,6$ & $0,4 \pm 3,6$ & 0,258 \\
\hline Colesterol (mg/1.000kcal) & $101,7 \pm 58,3$ & $105,8 \pm 65,0$ & $99,2 \pm 64,5$ & $112,8 \pm 61,7$ & 0,749 \\
\hline Fibras (g/1.000kcal) & $2,4 \pm 1,7$ & $2,3 \pm 2,2$ & $2,7 \pm 2,6$ & $1,9 \pm 1,3$ & 0,196 \\
\hline AGP/AGS & $0,22 \pm 0,14$ & $0,22 \pm 0,15$ & $0,19 \pm 0,14$ & $0,14 \pm 0,15$ & 0,079 \\
\hline
\end{tabular}

$\mathrm{AGS}=$ ácidos graxos saturados; $\mathrm{AGP}=$ ácidos graxos polinsaturados; $\mathrm{AGM}=$ ácidos graxos monoinsaturados .

* Valor P - Teste de Kruskal-Wallis para mais de duas amostras independentes.

** $A$ ingestão do álcool é apresentada entre os entrevistados que consumiram álcool.

Com o aumento da renda familiar, homens e mulheres apresentaram uma tendência de elevação do consumo de lipídios totais. Nas mulheres, esse aumento foi observado em todas as frações enquanto em homens ocorreu somente para a fração saturada.

O perfil alimentar apresentado se mostra ora semelhante, ora diferente àqueles descritos em países desenvolvidos e em desenvolvimento (Cervato, 1995; Drewnowski \& Popkin, 1997). Além disso, uma certa incongruência observada entre renda e escolaridade, principalmente dentro dos estratos de gênero e idade, sugere que os dois fatores podem estar diferencialmente relacionados ao perfil de consumo (Kushi et al., 1988).
A ingestão de energia nessa população foi consistentemente superior a outros estudos brasileiros (Cervato, 1995; Galeazzi et al., 1997). Quando estratificado por gênero, essa diferença persistiu somente entre homens, apresentando relação inversa com a idade. Mulheres bambuienses relataram consumo energético semelhante àquelas residentes em Campinas e em uma comunidade universitária (Anselmo et al., 1992; Galeazzi et al., 1997). O consumo energético associou-se à escolaridade; entretanto, parte desse efeito pode ser resultado da distribuição heterogênea dos níveis de escolaridade nos grupos etários.

A participação de carboidratos na energia total entre as mulheres foi proporcionalmente 
maior que entre os homens; porém, entre aquelas de maior poder aquisitivo, foi observado um decréscimo relevante na ingestão de carboidratos. Esses dados parecem ser consoantes com alguns estudos que demonstram um efeito diferenciado na dieta quando utiliza-se nível educacional e de renda (Kushi et al., 1988; Martins et al., 1994).

Em contraste, conforme descrito por Martins et al. (1994) e Thompson et al. (1992), foi observado um aumento proporcional do consumo de lipídeos com o aumento da renda familiar, independente do gênero, ultrapassando o limite máximo recomendado de $30 \%$ da energia total em cerca de um terço dos indivíduos
(OMS, 1990). As maiores inadequações foram observadas nos mais velhos (42\%); nos de maior escolaridade, com $43 \%$ para homens e $39 \%$ para mulheres e nos de maior renda (48\%).

Em relação às frações lipídicas, alguns agrupamentos puderam ser observados. Cerca de $58 \%$ dos homens com mais de 60 anos e $52 \%$ de mulheres e homens com maior renda familiar apresentaram ingestão de saturadas acima da recomendação máxima de $10 \%$ da energia total e da média nacional descrita em inquérito brasileiro de 1996 (Monteiro et al., 2000), que foi de $8,9 \%$. Quanto às polinsaturadas, a ingestão relatada foi menor que a recomendação internacional de 3 a 7\% da energia total (OMS, 1990), e in-

Tabela 4

Ingestão diária de nutrientes selecionados (mediana e desvio interquartílico), em proporção das calorias totais, de acordo com a renda familiar e gênero. Município de Bambuí, Minas Gerais, Brasil, 1996-1997.

\begin{tabular}{|c|c|c|c|c|}
\hline \multirow[t]{2}{*}{ Variáveis dietéticas } & \multicolumn{3}{|c|}{ Renda familiar (em salários mínimos) } & \multirow[t]{2}{*}{$p^{*}$} \\
\hline & $\leq 1,5$ & $3-5$ & $\geq 8$ & \\
\hline Mulheres $(n=304)$ & $n=91$ & $n=163$ & $n=50$ & \\
\hline Energia (kcal) & $2.752,1 \pm 1.491,3$ & $2.826,6 \pm 1.621,8$ & $2.818,9 \pm 1.463,4$ & 0,242 \\
\hline Carboidratos (\% kcal) & $59,2 \pm 15,4$ & $60,0 \pm 12,4$ & $55,5 \pm 10,1$ & 0,034 \\
\hline Proteínas (\% kcal) & $14,5 \pm 4,1$ & $14,6 \pm 2,8$ & $15,3 \pm 2,3$ & 0,577 \\
\hline Lipídios (\% kcal) & $26,3 \pm 12,6$ & $27,0 \pm 9,6$ & $29,4 \pm 8,3$ & 0,026 \\
\hline AGS (\% kcal) & $8,0 \pm 5,0$ & $5,8 \pm 3,3$ & $10,1 \pm 3,4$ & 0,001 \\
\hline AGP (\% kcal) & $1,7 \pm 1,1$ & $1,8 \pm 1,0$ & $2,2 \pm 0,8$ & 0,005 \\
\hline AGM (\% kcal) & $3,5 \pm 3,1$ & $3,7 \pm 2,6$ & $4,6 \pm 2,1$ & 0,049 \\
\hline \multicolumn{5}{|l|}{ Álcool (\%) } \\
\hline Usuárias & 38 & 60 & 70 & $<0,001$ \\
\hline Kcal** & $0,3 \pm 0,8$ & $0,3 \pm 0,8$ & $0,3 \pm 1,5$ & 0,300 \\
\hline Colesterol (mg/1.000kcal) & $89,2 \pm 68,9$ & $99,3 \pm 57,6$ & $104,5 \pm 56,2$ & 0,037 \\
\hline Fibras (g/1.000kcal) & $3,0 \pm 3,3$ & $3,1 \pm 2,6$ & $3,8 \pm 2,5$ & 0,184 \\
\hline AGP/AGS & $0,20 \pm 0,15$ & $0,22 \pm 0,11$ & $0,22 \pm 0,14$ & 0,905 \\
\hline Homens $(n=242)$ & $\mathrm{n}=50$ & $\mathrm{n}=130$ & $n=62$ & \\
\hline Energia (kcal) & $4.022,6 \pm 2.603,9$ & $3.681,9 \pm 2.299,1$ & $3.836,9 \pm 1.809,3$ & 0,374 \\
\hline Carboidratos (\% kcal) & $58,2 \pm 15,0$ & $56,6 \pm 14,6$ & $52,4 \pm 15,7$ & 0,236 \\
\hline Proteínas (\% kcal) & $15,6 \pm 4,6$ & $14,7 \pm 3,2$ & $15,0 \pm 4,1$ & 0,271 \\
\hline Lipídios (\% kcal) & $28,1 \pm 10,3$ & $26,3 \pm 11,8$ & $30,0 \pm 11,9$ & 0,031 \\
\hline AGS (\% kcal) & $9,5 \pm 4,8$ & $8,6 \pm 4,0$ & $10,3 \pm 5,4$ & 0,053 \\
\hline AGP (\% kcal) & $1,7 \pm 1,2$ & $1,5 \pm 0,8$ & $1,8 \pm 1,0$ & 0,018 \\
\hline AGM (\% kcal) & $4,0 \pm 2,5$ & $3,8 \pm 2,8$ & $4,3 \pm 2,5$ & 0,231 \\
\hline \multicolumn{5}{|l|}{ Álcool (\%) } \\
\hline Usuários & 54 & 71 & 79 & $<0,001$ \\
\hline $\mathrm{Kcal}^{\star \star}$ & $1.6 \pm \pm 2,8$ & $1,7 \pm 3,9$ & $1,2 \pm 3,2$ & 0,439 \\
\hline Colesterol (mg/1.000kcal) & $115,8 \pm 88,0$ & $99,4 \pm 50,4$ & $117,0 \pm 60,8$ & 0,093 \\
\hline Fibras (g/1.000kcal) & $2,2 \pm 2,5$ & $2,3 \pm 2,7$ & $2,7 \pm 2,1$ & 0,742 \\
\hline AGP/AGS & $0,23 \pm 0,18$ & $0,19 \pm 0,15$ & $0,20 \pm 0,13$ & 0,294 \\
\hline
\end{tabular}

AGS = ácidos graxos saturados; $A G P$ = ácidos graxos polinsaturados; $A G M=$ ácidos graxos monoinsaturados. * Valor P - Teste de Kruskal-Wallis para mais de duas amostras independentes.

** A ingestão do álcool é apresentada entre os entrevistados que consumiram álcool. 
Ingestão diária de nutrientes selecionados (mediana e desvio interquartílico), em proporção das calorias totais, de acordo com a escolaridade e gênero. Município de Bambuí, Minas Gerais, Brasil, 1996-1997.

\begin{tabular}{|c|c|c|c|c|}
\hline \multirow[t]{2}{*}{ Variáveis dietéticas } & \multicolumn{3}{|c|}{ Escolaridade (anos) } & \multirow[t]{2}{*}{$p^{*}$} \\
\hline & $\leq 4$ & $5-8$ & $8+$ & \\
\hline Mulheres $(n=304)$ & $n=163$ & $n=57$ & $\mathrm{n}=84$ & \\
\hline Energia (kcal) & $2.716,4 \pm 1.357,0$ & $2.489,5 \pm 1.953,6$ & $2.986,6 \pm 1.533,1$ & 0,004 \\
\hline Carboidratos (\% kcal) & $60,0 \pm 15,4$ & $58,3 \pm 13,0$ & $57,0 \pm 9,6$ & 0,289 \\
\hline Proteínas (\% kcal) & $15,0 \pm 3,4$ & $15,6 \pm 3,5$ & $14,9 \pm 2,7$ & 0,010 \\
\hline Lipídios (\% kcal) & $26,6 \pm 12,7$ & $27,4 \pm 8,0$ & $28,9 \pm 9,1$ & 0,245 \\
\hline AGS (\% kcal) & $8,6 \pm 5,0$ & $8,8 \pm 3,6$ & $9,3 \pm 3,3$ & 0,202 \\
\hline AGP (\% kcal) & $1,7 \pm 1,1$ & $1,9 \pm 0,9$ & $2,0 \pm 0,9$ & 0,009 \\
\hline AGM (\% kcal) & $3,7 \pm 2,8$ & $3,6 \pm 3,6$ & $4,2 \pm 2,4$ & 0,309 \\
\hline \multicolumn{5}{|l|}{ Álcool (\%) } \\
\hline Usuárias & 44 & 54 & 77 & $<0,001$ \\
\hline Kcal ${ }^{\star \star}$ & $0,3 \pm 0,6$ & $0,4 \pm 1,3$ & $0,3 \pm 1,0$ & 0,196 \\
\hline Colesterol (mg/1.000kcal) & $101,8 \pm 67,0$ & $88,7 \pm 49,9$ & $101,7 \pm 49,6$ & 0,167 \\
\hline Fibras (g/1.000kcal) & $3,0 \pm 2,7$ & $3,5 \pm 2,4$ & $3,5 \pm 3,5$ & 0,102 \\
\hline AGP/AGS & $0,21 \pm 0,15$ & $0,24 \pm 0,10$ & $0,22 \pm 0,13$ & 0,614 \\
\hline Homens $(n=242)$ & $n=132$ & $n=51$ & $n=59$ & \\
\hline Energia (kcal) & $3.711,9 \pm 2.310,6$ & $3.747,6 \pm 2.140,6$ & $4.019,5 \pm 1.833,1$ & 0,485 \\
\hline Carboidratos (\% kcal) & $56,7 \pm 15,1$ & $56,3 \pm 12,8$ & $53,0 \pm 16,6$ & 0,453 \\
\hline Proteínas (\% kcal) & $15,4 \pm 3,9$ & $14,6 \pm 3,1$ & $14,7 \pm 3,1$ & 0,205 \\
\hline Lipídios (\% kcal) & $27,0 \pm 9,7$ & $27,8 \pm 9,7$ & $27,4 \pm 13,4$ & 0,271 \\
\hline AGS (\% kcal) & $9,1 \pm 5,4$ & $8,6 \pm 4,1$ & $8,9 \pm 4,7$ & 0,839 \\
\hline AGP (\% kcal) & $1,5 \pm 1,1$ & $1,7 \pm 0,7$ & $1,6 \pm 1,0$ & 0,351 \\
\hline AGM (\% kcal) & $3,9 \pm 2,9$ & $4,2 \pm 2,0$ & $3,9 \pm 2,7$ & 0,948 \\
\hline \multicolumn{5}{|l|}{ Álcool (\%) } \\
\hline Usuários & 60 & 76 & 85 & $<0,001$ \\
\hline Kcal** & $1,3 \pm 2,3$ & $2,1 \pm 3,2$ & $1,3 \pm 4,4$ & 0,415 \\
\hline Colesterol (mg/1.000kcal) & $102,7 \pm 68,7$ & $99,2 \pm 46,9$ & $109,5 \pm 61,2$ & 0,903 \\
\hline Fibras (g/1.000kcal) & $2,3 \pm 2,3$ & $2,5 \pm 3,2$ & $2,5 \pm 1,4$ & 0,471 \\
\hline AGP/AGS & $0,20 \pm 0,16$ & $0,21 \pm 0,14$ & $0,20 \pm 0,15$ & 0,974 \\
\hline
\end{tabular}

AGS = ácidos graxos saturados; $A G P$ = ácidos graxos polinsaturados; $A G M=$ ácidos graxos monoinsaturados. * Valor P - Teste de Kruskal-Wallis para mais de duas amostras independentes.

** A ingestão do álcool é apresentada entre os entrevistados que consumiram álcool.

ferior aos achados de outros estudos brasileiros (Galeazzi et al., 1997; Monteiro et al., 2000). Vale ressaltar que, para todas as idades, faixas de renda e escolaridade, verificou-se que a ingestão de AGP apresentou proporções de inadequação que variou de $83 \%$ a $94 \%$ nessa população.

A ingestão de colesterol, via de regra, também excedeu o limite recomendado pela Organização Mundial da Saúde (OMS), de 300mg/ dia ou $100 \mathrm{mg} / 1.000 \mathrm{kcal}$, tanto em homens quanto em mulheres. Cerca de $50 \%$ da amostra apresentou excesso de consumo e, de novo, as maiores proporções estiveram concentradas diferencialmente entre os participantes de maior idade, renda e escolaridade. Estudos relataram que os homens aumentaram o consumo de co- lesterol e diminuíram o de carboidratos com a elevação da renda (Kushi et al., 1988; Shimakawa et al., 1994). O mesmo foi observado em Bambuí, porém apenas entre as mulheres.

As mulheres ingeriram relativamente mais fibras em comparação aos homens em todas faixas de idade, renda e escolaridade, apresentando uma leve tendência de aumento de acordo com a escolaridade, e uma redução significativa entre aquelas com mais de 60 anos. $\mathrm{O}$ consumo de fibras, embora abaixo das necessidades diárias, foi superior àquele encontrado em outros estudos (Kushi et al., 1988; van Horn et al., 1991). Entretanto, a ingestão de fibras em Bambuí não se associou à renda, como observado no estudo de Thompson et al. (1992). 
O consumo relativo de álcool pelos homens foi superior cerca de quatro vezes em cada faixa etária quando comparado com as mulheres, apresentando redução gradual em ambos os gêneros com a idade. A proporção de usuários de até trinta anos foi duas a três vezes maior que a de usuários acima de sessenta anos. A proporção de adeptos ao álcool nessa população aumentou com a elevação dos níveis de renda em ambos os gêneros, variando de $38 \%$ a $79 \%$. A mesma relação foi observada com a escolaridade, variando, no entanto, de $44 \%$ a $85 \%$.

Várias considerações merecem destaque neste estudo. Homens e mulheres apresentam perfis alimentares bastante diversos e essas diferenças se exacerbam quando a ingestão alimentar é estratificada por idade, renda e escolaridade. É interessante notar que mulheres apresentaram dieta mais saudável que os homens, fato também descrito em países europeus por Horldsworth et al. (2000).

Diferenças metodológicas adotadas nos diferentes estudos já realizados, bem como a heterogeneidade demográfica e sócio-econômica das populações estudadas, poderiam contribuir para explicar algumas divergências encontradas. Os instrumentos dietéticos utilizados, sua variabilidade intrínseca e extrínseca, questões relacionadas à amostragem e à distribuição assimétrica dos dados alimentares também podem contribuir para tais variações.

Problemas relativos ao instrumento utilizado poderiam ter contribuído para uma superestimação da ingestão. Porém, o ajustamento de nutrientes pelo total de ingestão calórica, com o objetivo de determinar o efeito ajustado do nutriente, possibilita contornar este problema. Várias estratégias de ajustes pela energia total são conhecidas. Destacam-se o método de densidade calórica obtida pela participação relativa do nutriente nas calorias totais e o método de resíduo do modelo de regressão, entre outros. Para estudos epidemiológicos de associação, o método de resíduo tem sido apontado como o mais apropriado, levando em conta sua capacidade de lidar de forma mais adequada com variáveis de confusão (Willet, 1998). A análise por densidade de nutriente, adotada nesta investigação, um estudo de perfil alimentar e não de associação, foi utilizada por ser um método simples, prático e possibilitar comparações com outros estudos (Fornes et al., 2000; Kushi et al., 1988; McNamara, 2000; Pereira \& Koifman, 1999; Willett, 1998).

A amostra trabalhada, considerando a adesão final (82,7\%) e a previsão para perdas (20\%) descrita por Lopes (1999), que não foram diferenciais, permite inferir um alto grau de validade interna possibilitando a generalização desses dados para a população de Bambuí.

Investigações realizadas nas capitais e regiões metropolitanas do país têm mostrado um padrão alimentar inadequado (menos saudável), com elevação dos lipídios totais, AGS e colesterol, além de uma redução de carboidratos complexos e de fibras na dieta dos brasileiros (Galeazzi et al., 1997; Monteiro et al., 2000). Os resultados de Bambuí também sugerem que o mesmo fenômeno pode estar também presente no interior do país. Nessa pequena cidade verificou-se, além das inadequações alimentares das grandes metrópoles, que a dieta mostrou-se hipercalórica e pobre em AGP e, portanto, com potencial de risco acrescido para doenças crônico-degenerativas, principalmente as cardiovasculares. Esses resultados são coerentes com aqueles descritos por Lima-Costa et al. (2001), mostrando alta prevalência de hipercolesterolemia e hipertensão arterial em Bambuí.

Portanto, os resultados de Bambuí, sob a ótica da distribuição etária, de renda familiar e escolaridade, contribuem para o conhecimento dos possíveis agregados de risco alimentar para a promoção de intervenções em saúde pública dirigidas a grupos específicos, com base em características da dieta global e não apenas em alimentos ou nutrientes isoladamente. Essa dimensão do estudo está em sintonia com a literatura atual, preocupada não só com a ingestão dietética relatada, mas também com o contexto nutricional, social e de comportamento (Fung et al., 2001; Pryer et al., 2001a, 2001b).

Novas investigações com a utilização do mesmo instrumento para a determinação do padrão alimentar são recomendadas, a fim de se verificar se os resultados do presente trabalho são generalizáveis para outras populações do interior do país. 


\section{Referências}

ANSELMO, M. A. C.; BURINI, R. C.; ANGELELT, A. Y. O.; MOTA, N. G. S. \& CAMPANA, A. O., 1992. Avaliação do estado nutricional de indivíduos adultos sadios, de classe média. Ingestão energética e protéica, antropometria, exames bioquímicos de sangue e testes de imunocompetência. Revista de Saúde Pública, 26:46-53.

BURGOS, M. G. P. A.; RIBEIRO, M. A.; MELO FILHO, S. C. \& CABRAL, P. C., 1996. Composição centesimal de ovo de galinha, carne de boi e de frango consumidos na área metropolitana de Recife, nordeste brasileiro. Revista de Nutrição da PUCCAMP, 9:224-235.

CERVATO, A. M., 1995. Dieta Habitual e Fatores de Risco para Doenças Cardiovasculares. Dissertação de Mestrado, São Paulo: Faculdade de Saúde Pública, Universidade de São Paulo.

DREWNOWSKI, A. \& POPKIN, B. M., 1997. The nutrition transition: New trends in the global diet. $\mathrm{Nu}$ trition Reviews, 55:31-43.

FORNES, N. S.; MARTINS, I. S.; HERNAN, M.; VELASQUEZ-MELENDEZ, G. \& ASCHERIO, A., 2000 Frequency of food consumption and lipoprotein serum levels in the population of an urban area, Brazil. Revista de Saúde Pública, 34:380-387.

FRANCO, G., 1995. Tabela de Composição Química dos Alimentos. 9a Ed. Rio de Janeiro: Editora Atheneu.

FUNG, T. T.; RIMM, E. B.; SPIEGELMAN, D.; RIFAI, N.; TOFLER, G. H.; WILLETT, W. C. \& HU, F. B., 2001. Association between dietary patterns and plasma biomarkers of obesity and cardiovascular disease risk. American Journal of Clinical Nutrition, 73:6167.

GALEAZZI, M. A. M.; DOMENE, S. M. A. \& SICHIERI, R., 1997. Estudo Multicêntrico sobre Consumo Alimentar e Estado Nutricional: Cadernos de Debate. Brasília: Instituto Nacional de Alimentação e Nutrição, Ministério da Saúde/Núcleo de Estudos em Alimentação, Universidade Estadual de Campinas.

HOLDSWORTH, M.; GERBER, M.; HASLAM, C.; SCALI, J.; BEARDSWORTH, A.; AVOLLONE, M. H. \& SHERRATT, E., 2000. A comparison of dietary behaviour in Central England and a French Mediterranean region. European Journal of Clinical $\mathrm{Nu}$ trition, 54:530-539.

KUSHI, L. H.; FOLSOM, A. R.; JACOBS, D. R.; LUEPKER, R. V.; ELMER, P. J. \& BLACKBURN, H., 1988. Educational attainment and nutrient consumption patterns: The Minnesota Heart Survey. Journal of the American Dietetic Association, 88:12301236.

LIMA-COSTA, M. F. F.; BARRETO, S. M.; UCHOA, E.; FIRMO, J. O. A.; VIDIGAL, P. G. \& GUERRA, H. L., 2001. The Bambuí Health and Ageing Study (BHAS): Prevalence of risk factors and preventive health-care use. Revista Panamericana de Salud Pública, 9:219-227.

LIMA-COSTA, M. F. F.; UCHOA, E.; GUERRA, H. L.; FIRMO, J. O. A.; VIDIGAL, P. G. \& BARRETO, S. M., 2000. The Bambuí Health and Ageing Study (BHAS): Methodological approach and preliminary results. Revista de Saúde Pública, 34:126135.
LOPES, A. C. S., 1999. Calibrando o Questionário Semiquantitativo de Freqüência Alimentar (QSFA) em um Inquérito Nutricional Populacional. Dissertação de Mestrado, Belo Horizonte: Faculdade de Medicina, Universidade Federal de Minas Gerais.

MARTINS, I. S.; MAZZILLI, R. N.; ALONSO NIETO, R.; ALVARES, E. D.; OSHIRO, R.; MARUCCI, M. F. N. \& CAJASUS, M. I., 1994. Hábitos alimentares aterogêncios de grupos populacionais em área metropolitana da região sudeste do Brasil. Revista de Saúde Pública, 28:349-356.

McNAMARA, D. J., 2000. Dietary cholesterol and atherosclerosis. Biochimica et Biophysica Acta, 1529: 310-320.

MONTEIRO, C. A.; MONDINI, L. \& COSTA, R. B. L., 2000. Mudança na composição e adequação nutricional da dieta familiar nas áreas metropolitanas do Brasil (1988-1996). Revista de Saúde Pública, 34:251-258.

OLIVEIRA, J. E. D., 1993. Análise e tendências. Alimentação, nutrição, desenvolvimento, economia, qualidade de vida. Alimentação e Nutrição, 5:145-52.

OMS (Organización Mundial de la Salud), 1990. Dieta, Nutrición y Prevención de Enfermidades Crónicas. Serie de Informes Técnicos 797. Geneva: OMS.

PEREIRA, A. R. \& KOIFMAN, S., 1999. Uso do questionário de freqüência na avaliação do consumo alimentar pregresso. Revista de Saúde Pública, 33:610-621.

PHILIPPI, S. T.; RIGO, N. \& LORENZANO, C., 1995. Estudo comparativo entre tabelas de composição química dos alimentos para avaliação de dietas. Revista de Nutrição da PUCCAMP, 8:200-213.

PINHEIRO, A. B. V.; LACERDA, E. M. A. \& BENZECRY, E. H., 1994. Tabela para Avaliação de Consumo Alimentar em Medidas Caseiras. 2a Ed. Rio de Janeiro: Produção Independente.

PRYER, J. A.; COOK, A. \& SHETTY, P., 2001a. Identification of groups who report similar patterns of diet among a representative national sample of British adults aged 65 years of age or more. Public Health Nutrition, 4:787-795.

PRYER, J. A.; NICHOLS, R.; ELLIOTT, P.; THAKRAR, B.; BRUNNER, E. \& MARMOT, M., 2001b. Dietary patterns among a national random sample of British adults. Journal of Epidemiology and Community Health, 55:29-37.

SICHIERI, R. \& EVERHART, J., 1998. Validity of a Brazilian food frequency questionnaire against dietary recalls and estimated energy intake. $\mathrm{Nu}$ trition Research, 18:1649-1659.

SHIMAKAWA, T.; SORIIE, P.; CARPENTER, M. A.; CAN, B.; DENNIS, B.; TELL, G. S.; WATSON, R. \& WILLIAMS, O. D., 1994. Dietary patterns and sociodemographic factors in the atherosclerosis risk in communities study. Preventive Medicine, 23:769-780.

SOARES, J. F. \& SIQUEIRA, A. L., 1999. Introdução à Estatística Médica. Belo Horizonte: Editora UFMG.

THOMPSON, F. E.; SOWERS, M. F; FRONGILLO, E. A. \& PARPIA, B. J., 1992. Sources of fiber and fat in diets of US women aged 19 to 50: Implications for nutrition education and policy. American Journal of Public Health, 82:695-702. 
VALENTE, F. L. S., 1997. Do combate à fome à segurança alimentar e nutricional: $\mathrm{O}$ direito à alimentação adequada. Revista de Nutrição da PUCCAMP, 10:20-36.

van HORN, V. L.; BALLEW, C.; LIU, K.; RUTH, K.; McDONALD, A.; HILNER, J. E.; BURK, G. L.; SAVAGE, P. J.; BRAGG, C. \& CAN, B., 1991. Diet, body, size, and plasma lipids-lipoproteins in young adults: Differences by race and sex. American Journal of Epidemiology, 133:9-23.

WILLETT, W. C., 1998. Nutritional Epidemiology. New York: Oxford University Press.

WILLETT, W. C.; HOWE, G. R. \& KUSHI, L. H., 1997. Adjustment for total energy intake in epidemiologic studies. American Journal of Clinical Nutrition, 65(Sup.):1220S-1228S.

Recebido em 8 de julho de 2002

Versão final reapresentada em 10 de março de 2003

Aprovado em 13 de junho de 2003 